\title{
Local corticosteroids, why are they not used more /
}

\section{properly?}

It has been almost 50 years since Niels Mygind published the first paper on the use of nasal corticosteroids. Twenty years ago, Weiner et al. showed in their systematic review that nasal corticosteroids are more effective than oral antihistamines in patients with allergic rhinitis.

Over 3000 papers later, all showing the efficacy and superiority of local corticosteroids over most other treatment options available in rhinitis and chronic rhinosinusitis (CRS); we still have not been able to convince the public and our colleagues that most patients with rhinitis/rhinosinusitis would significantly benefit from the use of a local corticosteroid.

In this issue, Jonas Jae-Hyun Park et al. show the embarrassing rates of nasal corticosteroid use in patients with CRS in Germany. In patients coded as having 'chronic sinusitis' nasal corticosteroids were prescribed at a very low rate (GP: $12.3 \%$, ENT: $34.3 \%)$. In patients coded as having 'nasal polyps' the numbers were slightly better. And unfortunately, Germany is not different from other countries; similar data concerning CRS has been published in the UK and Canada. Not only prescription is very limited, but actual use is even lower ${ }^{(1)}$. There is very little data in the literature on prescription rate in rhinitis and rhinosinusitis and surprisingly little data on ways to improve adherence.

Several barriers can impede the use of INCSs, including concerns about safety, misperceptions regarding the loss of response from frequent use, and undesirable sensations associated with intranasal administration. Caregivers, including otorhinolaryngologists, pulmonologist, GPs and pharmacists can help allay these concerns ${ }^{(2)}$. Furthermore, direct information with the use of digital tools and social media can better inform potential patients, including adolescents and (parents of) children ${ }^{(3,4)}$.
For CRS, and especially CRSwNP, it has been shown that other forms of application than nasal spray are probably more effective in reducing symptoms. In this issue of the Journal Reychler et al. systematically evaluated the clinical efficacy of intranasal drug delivery by nebulization in chronic rhinosinusitis and conclude that as things stand, this is not superior to nasal spray. However rinsing (with local corticosteroid) has been shown to be more effective than spray in CRS ${ }^{(5)}$, although in severe disease even rinsing does not seem to reach the sinuses ${ }^{(6)}$. Moreover, we know from clinical practice that compliance is an issue when asking patients to rinse their nose daily. The use of drug-eluting stents might overcome many of the difficulties involved with getting a high local dose of corticosteroids into the sinuses. We await drug-eluting stents that can be easily placed and disperse a high quantity of local corticosteroids over a long period of time without hampering safety ${ }^{(7)}$.

One of the first steps towards personalized medicine and endotyping in CRS might be using (absence of) eosinophils to predict response to local corticosteroids and/or macrolides ${ }^{(8)}$. In this issue Zeng et al. were not able to show differences in the efficacy of fluticasone propionate versus clarithromycin for postoperative treatment of CRSsNP and eosinophilic and noneosinophilic CRSwNP Chinese patients. But more studies will arrive to help us to find the right biomarker to predict response to treatment.

You will find many other topics of interest in the April issue, from the lack of systemic absorption of atomised intranasal cocaine during endoscopic sinus surgery, to the safety and feasibility of propofol sedation during Drug Induced Sedation Endoscopy (DISE) of the upper airway of patients with obstructive sleep apnea (OSA) without the presence of an anesthesiologist. Enjoy reading this second Rhinology issue of 2019!

\section{References}

1. Rudmik L, Xu Y, Liu M, Bird C, Kukec E, Quan H. Utilization Patterns of Topical Intranasal Steroid Therapy for Chronic Rhinosinusitis: A Canadian Population-Based Analysis. JAMA otolaryngology-head \& neck surgery. 2016;142(11):1056-62.

2. Bridgeman MB. Overcoming barriers to intranasal corticosteroid use in patients with uncontrolled allergic rhinitis. Integrated pharmacy research \& practice. 2017;6:109-19.

3. Bousquet J, Devillier P, Arnavielhe S, Bedbrook A, Alexis-Alexandre G, van Eerd M, et al. Treatment of allergic rhinitis using mobile technology with realworld data: The MASK observational pilot study. Allergy. 2018;73(9):1763-74
4. Seys SF, Bousquet J, Bachert C, Fokkens WJ, Agache I, Bernal-Sprekelsen M, et al. mySinusitisCoach: patient empowerment in chronic rhinosinusitis using mobile technology. Rhinology. 2018;56(3):209-15.

5. Harvey RJ, Snidvongs K, Kalish LH, Oakley GM, Sacks R. Corticosteroid nasal irrigations are more effective than simple sprays in a randomized double-blinded placebo-controlled trial for chronic rhinosinusitis after sinus surgery. International forum of allergy \& rhinology. 2018;8(4):461-70.

6. Aanaes K, Alanin MC, Nielsen KG, Moller Jorgensen M, von Buchwald C, Hoiby N, et al. The accessibility of topical treatment in the paranasal sinuses on operated cystic fibrosis patients assessed by scintigraphy. Rhinology. 2018;56(3):268-73

7. Hellings PW, Akdis CA, Bachert C, Bousquet J, Pugin B, Adriaensen G, et al. EUFOREA Rhinology Research Forum 2016: report of the brainstorming sessions on needs and priorities in rhinitis and rhinosinusitis. Rhinology. 2017;55(3):202-10.

8. Oakley GM, Christensen JM, Sacks R, Earls P, Harvey RJ. Characteristics of macrolide responders in persistent post-surgical rhinosinusitis. Rhinology. 2018.56 (2): 111-7.

Wytske J. Fokkens, Editor-in Chief Amsterdam, the Netherlands 